\title{
Genealogía y obtentores de la primera variedad de café (Coffea arabica L.) Catrenic seleccionada en Nicaragua
}

\section{Genealogy and breeders of the first Nicaraguan coffee (Coffea arabica L.) variety Catrenic selected in Nicaragua}

\begin{abstract}
Marisol Baylón Duarte
Ing. Agrónomo Fitotecnista. Maestrante Universidad Nacional Agraria de Nicaragua en agroecología y desarrollo sostenible. ORCID: https:// orcid.org/0000-0002-5967-032X / upolicidri_masatepe@yahoo.es
\end{abstract}

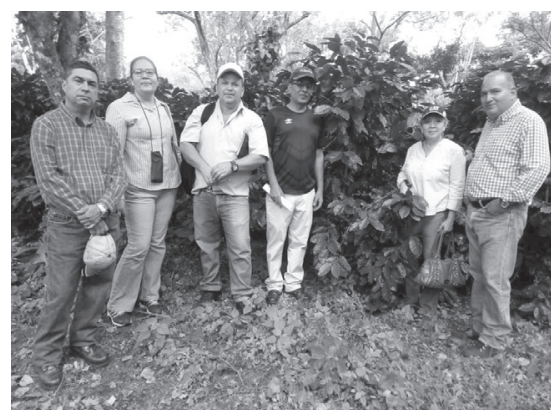

\begin{abstract}
RESUMEN
La roya del café (Hemileia vastratrix Berk \& Br.) fue identificada en Brasil en 1970; para enfrentar a este patógeno en Nicaragua, fueron introducidas en 1971, de la Estación de Introducción de Plantas de Glenn Dale, Maryland, Estados Unidos con código P.I.361030, al Jardín Botánico ubicado en Masatepe, Masaya, las primeras semillas de Catimor en generación $\mathrm{F}_{2}$. En este trabajo se utilizó el historial de las generaciones $\mathrm{F}_{2}$ hasta a la $\mathrm{F}_{6}$, que comprende los investigadores involucrados, ubicación, pasos, variables estudiadas, información basada en investigación realizada por investigadores antecesores y propia, documentada y publicada; hasta la obtención y registro de la variedad Catrenic. Se encuentran algunos datos relevantes de las variables evaluadas de la variedad en las diferentes generaciones (F), como los de producción promedio en la $\mathrm{F}_{4}$ que va de 2.166 a $3.995 \mathrm{~kg}$ uva por planta, estimándose una sobreproducción de 3.83 a $91.51 \%$ de acuerdo al testigo de mayor producción Catuaí amarillo con $2.086 \mathrm{~kg}$ uva por planta; la calidad de taza en la $\mathrm{F}_{5}$ de Catrenic en comparación con las variedades comerciales, no mostraron diferencias organolépticas significativas presentando los valores más altos a 900 m.s.n.m., obteniendo un valor de 2 en la escala de acidez, donde 3 es excelente, mientras que a una altura de 500 y 400 m.s.n.m. alcanzó un valor de 1. Finalmente, en la generación $\mathrm{F}_{6}$ se obtiene Catrenic, con excelente vigor híbrido, uniformidad fenotípica, porte intermedio, ramificación secundaria y terciaria (palmillas), entrenudos cortos, brote bronceado $\mathrm{y}$ verde, buena capacidad productiva (mayor que Caturra y Catuaí), tolerancia a roya, buena taza (como Caturra y Catuaí) y adaptabilidad a diferentes zonas. Esta variedad nicaragüense de café fue registrada legalmente en 2000 en el Departamento de Protección de Variedades del Registro de la Dirección de Semilla del Ministerio Agropecuario y Forestal.
\end{abstract}

Palabras clave: fitomejoramiento, Catimor, roya, Pacífico Sur, Centroamérica.

Recibido: 24 de junio del 2019

Aceptado: 10 de febrero del 2020

\begin{abstract}
The coffee rust (Hemileia vastratrix Berk \& Br.) was identified in Brazil in 1970, to face this pathogen in Nicaragua, they were introduced in 1971, from the Plant Introduction Station of Glenn Dale, Maryland, USA with code P.I.361030, to the Jardín Botanico located in Masatepe, Masaya, the first Catimor seeds in $F_{2}$ generation. In this work, the record of generations $F_{2}$ to $F_{6}$ was used, which includes the researchers involved, location, steps, variables studied, information based on research carried out by previous researchers and their own, documented and published; until obtaining and registering the Catrenic variety. There are some relevant data of the evaluated variables of the variety in the different generations (F), such as those of average production in the $\mathrm{F}_{4}$ that goes from 2,166 to $3,995 \mathrm{~kg}$ of berry per plant, estimating an overproduction of 3.83 to $91.51 \%$ according to the net plot of greater production $\mathrm{Y}$ short internodes, bronzed and green bud, good productive capacity (greater than Caturra and Catuaí), rust tolerance, good cup (similar Caturra and Catuaí) and adaptability to different areas yellow Catuaí with $2,086 \mathrm{~kg}$ of berry per plant; the cup quality in Catrenic's $F_{5}$ compared to commercial varieties, did not show significant organoleptic differences, presenting the highest values at 900 m.a.s.l., obtaining a value of 2 on the acidity scale, where 3 is excellent, while at a height of 500 and 400 m.a.s.l. reached a value of 1 . Finally, Catrenic is obtained in the $F_{6}$ generation, with excellent hybrid vigor, phenotypic uniformity, intermediate size, secondary and tertiary branching (splicing), short internodes, bronzed and green bud, good productive capacity (greater than Caturra and Catuaí), rust tolerance, good cup (similar Caturra and Catuaí) and adaptability to different areas. This Nicaraguan coffee variety was legally registered in 2000 in the Registry Variety Protection Department From the Seed Direction of the Ministry of Agriculture and Forestry.
\end{abstract}

Keywords: Plant breeding, Catimor, rust, South Pacific, Central America. 
$\mathrm{A}$ nte la amenaza que representaba la roya del café (Hemileia vastratrix Berk \& Br.), el Centro de Investigação das Ferrugens do Cafeeiro (CIFC) de Portugal "a finales de la década de los años 50, realizó una de las mayores contribuciones a la caficultura mundial mediante el descubrimiento del Híbrido de Timor (H.T). Desde entonces, sus investigadores postularon que se trataba de un híbrido natural probablemente entre Coffea arabica L. x Coffea canephora Pierre" (Alvarado, Moreno y Cortina 2002, p. 8), el que se mostró como "resistente a todas las razas de roya conocidas. Sin embargo, presenta características no deseables que imposibilitan su utilización en programas de mejora; por tanto, se han efectuado transferencias de la resistencia hacia las variedades comerciales de C. arabica" (Rivera y García, 1988).

Este híbrido, de acuerdo a Rodrigues (1990), "fue utilizado en el CIFC como progenitor preferencial para la producción de la resistencia en variedades comerciales; de ahí nacieron los derivados de H.T. (excepción hecha de la variedad Colombia)" (p. 209-210). Es importante conocer que "es en la Universidad Federal de Viçosa (UFV) en Brasil, que el material derivado del cruzamiento de Caturra por Híbrido de Timor e introducido a América Latina para ser ensayados (Rodrigues, 1990), a partir de la generación F3 se le denominó como Catimor (Rivera y García, 1988).

El mejoramiento genético en los cultivos perennes tiene como inconveniente el largo período de tiempo que lleva obtener una variedad; el café (Coffea arabica L.) en Nicaragua no ha sido la excepción por lo que en este trabajo se describen los pasos que se han seguido para conseguir la variedad "Catrenic que se adapta bien a nuestras condiciones" (Baylón y Zelaya, 1996, p. 48), su genealogía y obtentores, a partir de estas primeras semillas introducidas de café del material derivado del H.T. y por el tesonero trabajo de diferentes investigadores durante 30 años, quienes se convierten en obtentores, al ser las "persona físicas o morales que mediante un proceso de mejoramiento hayan obtenido y desarrollado, una variedad vegetal de cualquier género y especie" (SNICS, 2017). Ser obtentor es un derecho intransferible e imprescriptible.

\section{MATERIALES Y MÉTODOS}

Contexto de la investigación. Cuando aparece la roya en Brasil en 1970, las plantaciones de café en Nicaragua "estaban constituidas principalmente por variedades tradicionales de arábica susceptibles a roya, como Typica y Bourbon (Baylón y Pizzi, 1990), representando un problema fitosanitario de alto impacto para el que debían anticipar como enfrentarla, desarrollando variedades con resistencia o tolerancia al patógeno, que representara una opción para las zonas cafetaleras del país.
El trabajo de fitomejoramiento inicia en el año 1971 con la siembra de un híbrido introducido con genes de resistencia a roya en generación $F_{2}$, que por sucesivas generaciones de selección de las mejores plantas se logra estabilizar las características evaluadas en la generación $\mathrm{F}_{6}$, obteniendo la variedad Catrenic y registrándola en el año 2000 , con sus obtentores en un proceso que duró 30 años. La mayor parte del trabajo de investigación, validación y extensión se realizó en la zona cafetalera del pacífico sur de Nicaragua, que comprende los departamentos de Managua, Carazo, Masaya y Granada.

A continuación, se describe la metodología utilizada en el estudio, es el historial de las generaciones $\mathrm{F}_{2}$ hasta a la $\mathrm{F}_{6}$, que comprende los investigadores involucrados, ubicación, pasos, variables estudiadas, información basada en investigación realizada por investigadores antecesores y propia, documentada y publicada; hasta la obtención y registro de la variedad Catrenic.

Origen del material promisorio y período de estudio. De la Estación de Introducción de Plantas de Glenn Dale, Maryland, Estados Unidos, con el código P.I. 361030, se introdujo en semilla en 1971 al Jardín Botánico en Masatepe, Masaya, Nicaragua el primer derivado del H.T., con factores de tolerancia a roya del café en generación $\mathrm{F}_{2}$, con la finalidad de obtener un material productivo con resistencia (Baylón y Pizzi, 1993).

Este material recibido fue resultante de la hibridación artificial realizada en el año de 1959, entre el cafeto CIFC 19/1 Caturra rojo a partir de semillas proveniente de Angola y la planta CIFC 832/1 Híbrido de Timor, que originó el cruzamiento denominado CIFC Hw26 (Bettencourt, 1985).

\section{Antecedentes de las primeras investigaciones por antecesores \\ Generación $\mathbf{F}_{2}$. Establecida por el Ing. Agr. Miguel Bolaños} y el Agr. Manuel Aburto en un "banco de germoplasma constituido por un bloque, conformado de 20 plantas, tomando el código para su evaluación del bloque (en este caso 6), número de surco y número de planta. Se registran los datos individuales de plantas, de dos cosechas en los ciclos 1974/75 y 75/76 de producción café oro, relación uva:oro y porcentaje de flotación" (Bolaños y Aburto 1975).

Generación $\mathbf{F}_{3}$. Se integró un equipo de trabajo en el Programa de Ciencia y Tecnología, integrado por el Ing. Agr. Henry Matus, Rodolfo Munguía, Mario Herrera, Orontes Rosales y Marvin Solís y en el año 1977 seleccionaron las "mejores plantas, sembrando catorce descendencias en el Centro Experimental Campos Azules, Masatepe, con el código de CA del 1 al 14; aquí evaluaron adaptabilidad, 
desarrollo" (altura de planta, diámetro del tallo, número de bandolas, longitud de bandolas, número de nudos por bandola) “y producción” (Matus, Munguía, Herrera, Orontes y Solis, 1979).

\section{Antecedentes de investigaciones realizadas investigadores antecesores y base de datos propios Generación $\mathbf{F}_{4}$.}

Selección masal. Se eligieron los mejores materiales de la $\mathrm{F}_{3}$, conforme a los parámetros evaluados y "los establecieron en Campos de Observación en seis localidades ubicadas en Granada, Carazo y Masaya". Sin embargo, estos materiales fueron "producto de una selección masal por la mezcla de semilla de puntos muéstrales" (Matus et al., 1979). Además, en virtud de "diversas circunstancias en diferentes períodos no se hizo el manejo oportuno y adecuado a la mayoría de las parcelas, exceptuando la ubicada en el Centro Experimental en Masatepe y en Las Breñas, Jinotepe" (Baylón y Pizzi, 1993), por consiguiente, no se continuó con el registro de datos.

En 1980 el Agr. Gerardo Navarro establece en fincas, en diferentes zonas agroecológicas, incluyendo el Centro Experimental de Masatepe, seis progenies $\mathrm{F}_{4}$ en campos de observación y selección. En esta etapa se hace un alto en la investigación, para redefinir la metodología de fitomejoramiento.

Método genealógico o por pedigree (de aquí en adelante). En "1981, el Agr. Navarro junto con el Ing. Agr. Noel Dávila reordenan los procedimientos de fitomejoramiento, estableciendo la selección individual. Regresan a las 14 descendencias $\mathrm{F}_{3}$ establecidas en Campos Azules, Masatepe; cosechan las mejores plantas considerando los datos de producción, el porcentaje de flotación y relación uva-oro" (Baylón y Pizzi, 1988). En 1982 se establecen un Campo de Observación y Selección (COS) en el Centro Experimental de Café Mauricio López Munguía en Masatepe y lo continúa trabajando el Agr. Navarro.

Mediante el PROMECAFE (Programa para el Mejoramiento de la Caficultura de la Región), en el año 1985, como "parte del plan de visita a Centroamérica, se recibe la asesoría del Dr. Aníbal Jardim Bettencourt investigador principal del CIFC, sobre los temas de las razas fisiológicas de la roya, fuentes de resistencia y transferencia de genes de resistencia a variedades comerciales de café. El Dr. Bettencourt realizó una evaluación de los progresos alcanzados en la ejecución de los programas de mejoramiento genético y la situación de la roya del café" (Ramírez, 2017), además visitó los proyectos de investigación en ejecución con variedades resistentes entre ellos el COS en $\mathrm{F}_{4}$ ubicado en el Centro Experimental de café en Masatepe (también conocido como Jardín Botánico), donde observa un material vigoroso con una gran uniformidad fenotípica, revisa los registros de producción y la metodología, valorándolo como un material sobresaliente al que se le debe poner nombre. En este mismo año comienza a trabajar con el Agr. Navarro, la Ing. Agr. Marisol Baylón.

En el año 1986 finaliza el trabajo del Agr. Navarro y continúa la Ing. Baylón junto con el asistente Agr. Wilfredo Pizzi quien comienza a trabajar en el año 87, también se sigue contando con el asesoramiento del Instituto Interamericano de Cooperación para la Agricultura - Programa Cooperativo Regional para el Desarrollo Tecnológico y Modernización de la Caficultura (IICA-PROMECAFE) a través del Ing. Jorge Hernán Echeverri; de este COS F4 (también nombrado como el lote de La Torre), ubicado en el Jardín Botánico en Masatepe, se descartan las plantas que no cumplen con los parámetros esperados y "se seleccionaron las siete mejores plantas de acuerdo a los parámetros evaluados de Producción kg uva por planta, vigor, porcentajes de tipos de semillas tomados en 100 granos (fruto vano, plano, caracol, triángulo y monstruo), afección por problemas fitosanitarios (observaciones) y que se reflejan en los datos registrados de seis ciclos de cosecha acumulada por planta de 1985/86 a 90/91" (Baylón, Pizzi y Navarro, 1992).

De aquí en adelante se lleva el registro y análisis de información generada (datos por planta): Producción $\mathrm{kg}$ uva por planta y fechas de recolección, altura en metros, vigor (calificación subjetiva del 0 al 10 , tomando como modelo el cultivar caturra), porcentajes de tipos de semillas tomados en 100 granos (fruto vano, plano, caracol, triángulo y monstruo), tamaño promedio de tamiz y porcentaje de retención de zaranda, datos climatológicos, afección por problemas fitosanitarios (observaciones).

Generación F5. Se estableció en COS las progenies seleccionadas, usando códigos de surcos y número de plantas; "sembrándose en el Centro Experimental de Café del Pacífico en Masatepe y en fincas de productores colaboradores en otras condiciones agroecológicas de Masaya, Carazo y Managua (El Crucero), ubicadas en rangos de altitud que van de 454 a 900 msnm" (Baylón et al., 1992), igualmente "se envió semilla al fitomejorador Armando Vindel en el Centro Experimental de Café del Norte ubicado en Bonetillo, Jinotega, Nicaragua" (Baylón y Pizzi, 1993). En estos COS se comparó con las variedades comerciales Caturra, Catuaí y Pacas (Baylón et al., 1992), y se registran diferentes observaciones fitosanitarias y de comportamiento.

En esta etapa, se realizaron "pruebas de calidad de taza de Catrenic en comparación con las variedades comerciales, a altitudes de 400, 500 y 900 msnm" (Baylón y Guharay, 2001). Además, es "evaluado para resistencia a nematodos" (Rosales, Calderón y García, 1993). En 1991 es el último año que trabaja el asistente Agr. Pizzi permaneciendo la Ing. Agr. Baylón en el trabajo de fitomejoramiento. 
Generación F6. Se plantaron 19 líneas seleccionadas continuando con el trabajo en COS en fincas de productores colaboradores de Masatepe, Jinotepe, San Marcos, Las Esquinas, igualmente se envió semilla a la Ing. Patricia Contreras, Directora del CEN (Centro Experimental del Norte). En el Centro Experimental de Café del Pacífico (Jardín Botánico) se establecieron bancos de selección y multiplicación de semilla para abastecer a los productores.

Registro de la variedad Catrenic. En el año 2000 se realizaron los trámites correspondientes por el Ing. Agr. Sergio Obregón Vicegerente Técnico de la Unión Nicaragüense de Cafetaleros (UNICAFE) de quien dependía el Centro Experimental de Café, con el Ing. Agr. César Boza, Director General de Semillas del Ministerio Agropecuario y Forestal (MAG-FOR) para el registro de la variedad en el Departamento de Protección de Variedades del Registro.

La información necesaria para su debido registro, fue elaborada por la Ing. Agr. Marisol Baylón, incluyendo su trabajo de investigación de 17 años, así como la de los otros investigadores.

\section{RESULTADOS Y DISCUSIÓN \\ Evaluaciones realizadas desde la generación $F_{2}$ hasta la generación $F_{6}$, obtención de variedad Catrenic y registro.} Se presentan los resultados obtenidos en las selecciones de cada una de las generaciones, que tienen continuidad hasta conformar la variedad Catrenic.

Generación F2. De los registros documentados en las 20 plantas establecidas del Banco de germoplasma en dos "cosechas de los ciclos 1974/75 y 75/76 en la generación $F_{2}$, se obtuvo una gran variabilidad en cuanto a producción que va de 334.25 a $2410.40 \mathrm{~kg} \mathrm{ha}^{-1}$ de café oro, en cuanto a porcentaje de fruto vano de 12.28 a $50.50 \%$ y relación uva oro de 4.11:1 a 7.02:1 (Bolaños y Aburto 1975).

Generación $\mathbf{F}_{3}$. En el Centro Experimental Campos Azules, Masatepe, con el código de CA del 1 al 14, se obtienen registros que presentaron una gran variabilidad de todos los parámetros medidos en cuanto a altura de planta, diámetro del tallo, número y longitud de bandolas, numero de nudos por bandola y producción, solo cuatro resultaron estadísticamente iguales (Matus et al., 1979). No obstante, se obtuvieron datos relevantes de la cosecha de las mejores plantas de producción de 3000 a $3600 \mathrm{~kg} \mathrm{ha}^{-1}$, el porcentaje de flotación de $3 \%$ a 6.8\% y relación uva-oro de 5.04:1 a 6.61:1, discriminando los otros materiales por no considerarse sobresalientes (Baylón y Pizzi, 1988).

\section{Generación $\mathbf{F}_{4}$}

Selección masal. De los materiales establecidos en campos de observación en seis localidades ubicadas en Granada,
Carazo y Masaya, se obtuvieron datos, pero como "fueron producto de una selección masal por la mezcla de semilla de puntos muéstrales" (Matus et al., 1979), no forman parte del trabajo de selección. También las "seis progenies $\mathrm{F}_{4}$ sembradas en campos de observación en 1980 en fincas de diferentes zonas agroecológicas, son tomadas para la continuidad del trabajo. Algunos "productores observan el potencial y obtienen semilla sin estar estabilizado el material, por considerarla promisoria y asumiendo que sería liberado" (Detrinidad, 2004). Este material sin estabilidad.

Método genealógico o por pedigree. Al valorar la opinión del Dr. Bettencourt respecto a su apreciación sobre el híbrido en estudio, el Ing. Agr. Henry Matus quien funge como Director Nacional de Café en 1985, nombra a este material promisorio como Catrenic que significa "Catimor Revolución Nicaragua", al que aún no se le considera variedad, pues muestra variabilidad en producción, porcentaje de fruto vano y grano caracol. Aunque presenta uniformidad fenotípica en porte, forma de copa, largo de bandolas y empalmillamiento entre otros. En observación sin afectación de roya, afectación de minador (Leucoptera coffeella Guer), tolerancia al paloteo, excelente vigor.

Los datos registrados de seis ciclos de cosecha acumulada por planta de 1985/86 a 90/91, muestran variabilidad en los parámetros evaluados y mencionados anteriormente, pero se "seleccionan 7 mejores plantas que su producción promedio va de 2.166 a $3.995 \mathrm{~kg}$ uva por planta, estimándose una sobreproducción de 3.83 a $91.51 \%$ de acuerdo al testigo de mayor producción Catuaí amarillo con $2.086 \mathrm{~kg}$ uva promedio por planta; el fruto vano varió de $3 \%$ a $7 \%$ y el testigo $6 \%$, el grano plano de 72 a $82 \%$, siendo estos algunos de los datos registrados que se tomaron en cuenta para la selección de estos materiales" (Baylón, et al., 1992).

Generación F5. Los materiales establecidos en COS "en Masatepe y en fincas de productores colaboradores de Masaya, Carazo y Managua, ubicadas en rangos de altitud entre 454 y 900 msnm" (Baylón et al., 1992), superan "en producción a las variedades comerciales Catuaí, Caturra y Pacas" (Baylón, 2001), presenta "porte medio y uniformidad fenotípica" (Baylón y Zelaya, 1996), "buen vigor y tolerancia a roya, tiene la particularidad de producir palmilla, es uno de los materiales que ha mostrado mejor comportamiento" (Baylón, 1995).

En los datos registrados se destaca los obtenidos en El Crucero, Managua en cuanto al tamaño promedio de tamiz que osciló según los resultados de las "medias de 17.3 a 18 en la prueba de granulometría, donde los tamices predominantes fueron el $\mathrm{N}^{\circ} 17$ y $\mathrm{N}^{\circ} 18$, en los cuales se retuvo de 77.9 a $89.2 \%$ respectivamente, comparado con el cultivar regional 
Catuaí amarillo de tamaño promedio de tamiz de 17.5 donde el tamiz predominante fue el $\mathrm{N}^{\circ} 17$, con un porcentaje de retención de zaranda de 17/64 de 83\%" (Baylón et al., 1992); también se observó que conforme aumenta la altitud, el tamaño del grano tiende a aumentar.

En las pruebas de calidad de taza de Catrenic en comparación con las variedades comerciales, "no mostraron diferencias organolépticas significativas presentando los valores más altos a 900 msnm, obteniendo un Cuadro 1. Resumen genealógico y obtentores de la varieda d Catrenic valor de 2 en la escala Generación de acidez, donde 3 es excelente, mientras que a una altura de 500 y 400 msnm alcanzó un valor de 1" (Baylón y Guharay, 2001), presentando un "comportamiento similar en cuanto a altura en el tamaño promedio de tamiz" (Baylón y Pizzi, 1993). Además, fue evaluado para resistencia a nematodos junto con otros materiales, "resultando susceptible" (Rosales et al., 1993).

Generación $\quad$ F6. $_{\text {. }}$ Las 19 líneas seleccionadas mostraron su potencial productivo y tolerancia a roya, siendo de gran aceptación, los bancos de selección y multiplicación de semilla para abastecer a los productores establecidos en el Jardín Botánico de Masatepe, son inscritos en la Dirección de Semillas y se produce semilla certificada. Es en las generaciones $F_{5} \circ F_{6}$, que se han estabilizado las características fenotípicas, motivo del programa de mejoramiento; se establecen parcelas individuales por cada una de las progenies seleccionadas. La semilla de estas parcelas constituye la denominada "semilla básica", a las que se les realizaron pruebas de adaptabilidad en diversos ambientes cafetaleros, se evaluó el comportamiento agronómico, productivo, calidad de taza y fitosanitario. certificada.
Registro de la variedad. La variedad Catrenic fue registrada en el 2000 en el Departamento de Protección de Variedades del Registro de la Dirección de Semilla del MAG-FOR; propiedad intelectual de todos los involucrados, según su participación (Cuadro 1). Finalmente fue liberada en el año 2000, como variedad comercial a disposición de los productores.
$\mathrm{F}_{3} \quad$ Registro de datos individual de plantas Selección masal mezcla de semilla de puntos muestrales.

Selección individual.

Selección individual en Campo de Marisol Baylón

Wilfredo Pizzi

Marisol Baylón

Wilfredo Pizzi

Marisol Baylón Obtentor Año Centro de Investigação das Ferrugens do 1959 Cafeeiro (CIFC) de Portugal Introducción (P.I.361030) 1971 Miguel Bolaños y Manuel Aburto $1974-1976$ Henry Matus, Rodolfo Munguía, Mario 1977-1979 Herrera, Orontes Rosales y Marvin Solis

Paro de labores Gerardo Navarro y Noel Dávila

1978 $1980-1982$

$1983-1986$

$1985-1993$

$1987-1993$

1987- 1995

$1987-1991$

$1995-2000$

\section{CONCLUSIONES}

Primera variedad seleccionada en Nicaragua, un Catimor de nombre Catrenic, obtenida por el Método de Selección Genealógica o Pedigree, desde la generación $F_{2}$ hasta la $\mathrm{F}_{6}$, consolidando 30 años de investigación. En producción, supera a las variedades comerciales Catuaí, Caturra y Pacas, buen vigor y tolerancia a roya, produce palmilla, buen comportamiento, buena calidad de taza semejante a Catuaí, Caturra y Pacas. Se establecieron bancos de semilla

Variedad registrada en el año 2000, siendo propiedad intelectual de todos los investigadores involucrados, según su participación y liberada como variedad comercial a disposición de los productores. 


\section{REFERENCIAS BIBLIOGRÁFICAS}

Alvarado, G., Moreno, L., y Cortina, H. (2002). Caracteres agronómicos y resistencia incompleta a Hemileia vastatrix, de progenies de Caturra x Híbrido de Timor. Cenicafé 53(1),7-24.

Baylón, M. (1995). Variedades de café en Nicaragua. Masatepe, Nicaragua. Centro Experimental de Café del Pacífico Sur.

Baylón, M. (2001). Informe de avances del departamento de fitomejoramiento. Masatepe, Nicaragua. Centro Experimental de Café Jardín Botánico. UNICAFE.

Baylón, M., y Guharay, F. (2001). Curriculum para manejo de la calidad de café. Capacitación a capacitadores. Masatepe, Nicaragua. UNICAFE - CATIE MIP.

Baylón, M., y Pizzi, W. (1988). Avances del programa de fitomejoramiento. Masatepe, Nicaragua. VII Reunión Regional de Mejoramiento de Café. IICA PROMECAFE. San Salvador, El Salvador.

Baylón, M. y Pizzi, W. (1990). Avances del departamento de fitomejoramiento. Masatepe, Nicaragua. IX Reunión regional de mejoramiento de café. IICA PROMECAFE. Managua, Nicaragua.

Baylón, M. y Pizzi, W. (1993). Línea Catrenic (Coffea arabica L. Var. Catimor). Informe Departamento Fitomejoramiento. Masatepe, Nicaragua. Centro Experimental del Café del Pacífico "Jardín Botánico" CONCAFE.

Baylón, M. y Pizzi, W. (1993). Línea Catrenic (Coffea arabica L. Var. Catimor). XVI Simposio sobre caficultura latinoamericana. Volumen I. IICA - PROMECAFE. Managua, Nicaragua.

Baylón, M., Pizzi, W., y Navarro, G. (1992). Evaluación de la adaptabilidad de la línea Catrenic. XV Simposio Latinoamericano de Cafeticultura. IICA - PROMECAFE, Instituto Mexicano del Café, SARH. Veracruz, México.

Baylón, D., y Zelaya, D. (1996). Variedades de café en Nicaragua. Manuel de Caficultura de Nicaragua. (1.a ed., p 48). Managua. Unión Nicaragüense de Cafetaleros (UNICAFE).

Bettencourt, A. J. (1985). Características agronómicas de selecciones derivadas de cruzamientos entre el Hibrido de Timor y las variedades Caturra, Villasarchi y Catuaí. Boletín Promecafé No. 28 julio-septiembre.

Bolaños, M. y Aburto, M. (1975). Informe de 1974/75. Jardín Botánico, Masatepe, Nicaragua. Instituto Nicaragüense de Tecnología Agropecuaria (INTA).

Detrinidad, R. (2004). A la sombra del café. Reflexiones de un cafenómano. Managua, Nicaragua. Imprimatur Artes Gráficas.

Matus, H., Munguía, R, Herrera M., Orontes C., Solis, M. (1979). Primera evaluación de características agronómicas de 23 progenies provenientes de tres cultivares con resistencia a la roya del cafeto. II Simposio latinoamericano sobre caficultura. IICA PROMECAFE. Xalapa, México.

Ramírez, J. (2017). Variedades pierden resistencia a la roya en Centroamérica. ¿Qué aspectos son prioritarios para abordar el problema? Comunicaciones Técnicas de Café. Número 76. Recuperado de https://www.ramirezcaficulturadesdecostarica.com/ ct-76

Rivera, A., y García, L. (1988). Avances en la selección de progenies Catimor serie 9000 en México. VII Reunión Regional de Mejoramiento de Café. IICA PROMECAFE. San Salvador, El Salvador.

Rodrigues, C. (1990). La resistencia genética a la roya del cafeto. 50 años de Cenicafé 1938-1988: Conferencias conmemorativas. Chinchiná, Colombia. Federación de cafetaleros de Colombia, Centro nacional de investigaciones de café.

Rosales, J., Calderón, M., y García, P. (1993). Búsqueda de fuentes de resistencia en germoplasma de cafeto a nematodos fitoparásitos. II Congreso nacional de café. Avances técnicos enero - noviembre. CATIE/MAG-MIP. Managua, Nicaragua.

Servicio Nacional de Inspección y Certificación de Semillas (SNICS). (2017). ¿Qué es un obtentor? Servicio Nacional de Inspección y Certificación de Semillas. Gobierno de México. Cd. México, México. https://www.gob.mx/snics/articulos/que-es-unobtentor?idiom $=$ es 